\title{
ORTHOGONAL BLOCK CODE DESIGN FOR FREQUENCY-SELECTIVE MULTIPIE ANTENNA CHANNELS
}

\author{
Yanwu Ding, Timothy N. Davidson and K. Max Wong \\ Department of Electrical and Computer Engineering \\ McMaster University, Hamilton, Ontario, Canada
}

\begin{abstract}
Orthogonal Space-Time Block Codes (OSTBCs) have several desirable properties for delay limited communication over a coherent narrowband (aat) fading multiple antenna channel. In particular, they provide full diversity, and maximum likelihood detection can bc achieved via linear processing and symbol-by-symbol detection. As a result, the maximal symbol rate and minimal latencies of OSTBCs have been well studied. In this paper, we construct orthogonal block codes for frequency-selective multiple antenna channels, and study their symbol rate and latency properties. Our code construction is based on direct application of the orthogonality constraints. This enables us to show that the maximum symbol rate of an orthogonal block code for a chantel with memory length $L$ is $1 / L$ times the maximum rate of an OSTBC for the same antenna confguration. Orthogonal codes that achieve this maximal rate can be simply constructed via the Kronecker product of a rate maximal OSTBC with an identity matrix of size $L$. This Kronecker design scheme can also be applicd to construct onthogonal block codes for frequency-selective channels with other desirable properties, such as minimal latency.
\end{abstract}

\section{INTRODUCTION}

Orthogonal space-time block coding is a popular transmission scheme for narrowband coherent multiple antenna wireless links. Two key properties of the family of Orthogonal Space-Time Block Codes (OSTBCs) are that they enable full diversity gain, and that maximum likelihood detection can be achieved by linear processing and symbol-by-symbol detection. Several design schemes for OSTBCs have been established $[1,6,10,12-14]$, and the limits that the orthogonality constraint places on the maximum achievable (symbol) rate and the minimum achievable latency are well understood. There have been several approaches proposed for extending OSTBCs to frequency selective channels $[3-5,7-9,11,15]$. These designs typically combine existing OSTBCs with certain transmission techniques for single-input single-output frequencyselective channels, such as Orthogonal Frequency Division Multiplexing (OFDM) or the time-reversal transmission scheme.

The goal of the present paper is obtain a direct construction of orthogonal space time block codes for frequency-selective multiple antenna channels, so that all the desirable properties of OSTBCs can be extended to wideband transmission systems. We seek a defnitive statement of the limits that the orthogonality constraint places on the symbol rate and the latency of the code. We derive our construction using a "irst principles" approach in which we directly enforce the orthogonality conditions. This enables us to show that the orthogonality constraint can be decoupled into independent orthogonality constraints in the space and delay domains. That allows us to show that the maximum symbol rate of any orthogonal block code for a frequency-selective channel with memory length $L$ is $1 / L$ times the maximal OSTBC rate for the aat-fading case with the same antenna confguration. Orthogonal codes which achieve that symbol rate can be simply constructed via the Kronecker product of a rate-maximal OSTBC for the aatfading scenario $[1,6,12]$ with an identity matrix of size $L$. The Kronecker coding scheme is also valid for non-maximal-rate designs, such as minimal latency codes [14]. If the OSTBC used in the Kronecker product operation has the minimum latency. then the resulting orthogonal code for the frequency-selective channel also has minimum latency.

To place our construction in context, we point out that several schemes have been proposed for applying OSTBCs to frequencyselective multiple antenna channels. A standard approach is to incorporate the principles of orthogonal frequency division multiplexing (OFDM), such as the space-time/space-frequency OFDM (ST/SF-OFDM) schemes [5], and the circulant generalized delay diversity (CGDD) scheme [3]. These schemes obtain a symbol rate which is a factor of $T /(T+(L-1))$ lower than that of the corresponding OSTBC, where $T$ is the length of the data block. For long block lengths this factor can be close to 1 , but if low latency is required the block length will be short and the effect of the cyclic prefx on the symbol rate will be signifcant. Furthermore, many of these coding schemes do not provide full diversity, because the channel matrix may drop rank at some subcarrier frequencies. An alternative technique is the time-reversal space-time block coding (TR-STBC) scheme [4,9]. Its symbol rate reduction from the OSTBC case is a factor of $T /(T+2(L-1))$, and it also requires a more complex receiver. The TR-STBC scheme lies within a signifcantly larger class of coding schemes for frequencyselective multiple antenna channels [15]. The symbol rate reductions of the schemes in that class are of a similar order to those above, and some of the schemes provide full diversity, but the (optimal) detection problem remains substantially more expensive than that of OSTBCs in bat fading.

It is well known that the price paid for the advantages of orthogonality in the aat-fading environment is a reduction in the maximum (symbol) rate of the system. That is. OSTBCs provide a particular trade-off between rate. diversity (BER performance), and system complexity'. In this paper, we show that in the case of frequency-selective channels, the rate penalty for orthogonality is $L$ times larger than that for the dat-fading case. In spite

\footnotetext{
${ }^{1}$ Different coding schemes produce different trade-offs. For example, full-rate full diversity designs are available $[2,8]$, but they require a more complex receiver than an orthogonal design
} 
of this. simulation results show that the orthogonal block code for frequency-selective channels can provide better performance than some existing designs, such as the ST/SF-OFDM $[4,5]$ and CGDD [3] schemes.

The notations used in this paper are reasonably standard: Bold upper case letters denote matrices, $\boldsymbol{A}_{j}$ is a submatrix of $\boldsymbol{A}$, and $\boldsymbol{A}_{i j}$ is a submatrix of $\boldsymbol{A}_{j}$. Bold lower case letters denote column vectors, $\boldsymbol{a}_{j}$ is the $j$ th column in $\boldsymbol{A}, \boldsymbol{a}_{i, j}$ is the $i$ th column in $\boldsymbol{A}_{j}$. The vector vec $(A)$ is formed by stacking the columns of $A,(\cdot)^{T}$ denotes transpose. $(\cdot)^{H}$ denotes the conjugate transpose, and $I_{K}$ denotes the identity matrix of size $K$. The symbol $\otimes$ denotes the Kronecker product.

\section{SYSTEM MODEL}

We consider a multiple antenna communication system with $M$ transmit and $N$ receive antennas. The channel between the $j$ th transmit and $i$ th receive antenna is fregtency-selective with transfer function $H_{j i}(z)=\sum_{\ell=0}^{L-1} h_{j i}^{\ell} z^{-\ell}$. We assume that the coeffcients $\left\{h_{j i}^{\ell}\right\}$ are independent zero-mean complex circular Gaussian random variables with equal variance. The realization of $\left\{h_{j i}^{t}\right\}$ is assumed to be constant over a block and known at receiver but not known at transmitter. The noise is zero-mean Gaussian and statistically independent among the $N$ receive antennas. We consider a block transmission system and wc assume that at least $(L-1)$ zeros are padded between consecutive transmitted blocks in order to avoid inter-block interference at the receiver. The transmitted signal from the $j$ th transmit antenna over $(T+L-1)$ time slots is denoted by $s_{j}=\left(\begin{array}{llll}s_{1, j} & s_{2, j} & \cdots & s_{T+L-1, j}\end{array}\right)^{T}$, where $s_{T+1, j}=\cdots=s_{T+L-1, j}=0$. The received signal vector at the $i$ th receive antenna is

$$
\boldsymbol{y}_{i}=\sum_{j} \boldsymbol{H}_{j i} s_{j}+\boldsymbol{w}_{i}=\boldsymbol{H}_{i} s+\boldsymbol{w}_{i}
$$

where $\boldsymbol{y}_{\boldsymbol{i}}=\left(\boldsymbol{y}_{1, i} \cdots \boldsymbol{y}_{(T+1-L-1), i}\right)^{T}$, and $\mathcal{H}_{i}=$ $\left(\begin{array}{lll}\mathcal{H}_{1 i} & \cdots & \mathcal{H}_{M i}\end{array}\right)$, where $\mathcal{H}_{j i}$ is the $(T+L-1) \times 7$ lower triangular Toeplitz channel matrix between the $j$ th transmit antenna and the $i$ th receiver antenna. Its frst column is equal to $\left(\begin{array}{lllll}h_{j i}^{0} & \cdots & h_{j i}^{L-1} & 0 \cdots 0\end{array}\right)^{T}$. The vector $s=$ $\left(\begin{array}{lll}s_{1}^{T} & \cdots & s_{M}^{T}\end{array}\right)^{T}$, stacks all the signals transmitted from the $M$ transmit antennas, and $\boldsymbol{w}_{i}$ is the noise vector at the $i$ th receive antenna. Our goal is to design orthogonal block codes for the system in (1). To do so we need to reformulate (1) such that the signal transmission matrix for the system is explicit:

$$
\boldsymbol{y}_{i}=\sum_{j} \boldsymbol{S}_{j} h_{j i}+w_{i}=S h_{i}+w_{i}
$$

where $S=\left(\begin{array}{llll}S_{1} & S_{2} & \cdots & S_{M}\end{array}\right)$, and $S_{j}$ is the $(T+L-1) \times L$ Toeplitz. signal matrix transmitted from the $j$ th transmit antenna.

$$
S_{j}=\left(\begin{array}{cccc}
s_{1} j & 0 & \cdots & 0 \\
s_{2 j} & s_{1 j} j & \cdots & 0 \\
\vdots & & \ddots & \vdots \\
s_{T j} s_{(T-1)} & \cdots & s_{(T-C+1) j} \\
0 & s_{T} T^{\prime} j & \cdots & s_{(T-L) j}(T-L) \\
\vdots & & \ddots & \vdots \\
0 & 0 & \cdots & s_{T j} j
\end{array}\right) .
$$

The vector $h_{j i}=\left(\begin{array}{lll}h_{j i}^{0} & \cdots & h_{j i}^{L-1}\end{array}\right)^{T}$ contains the channel coeffeients from the $j$ th transmit antenna to the $i$ th receive an- tenna, and $h_{i}=\left(\begin{array}{lll}h_{1 i}^{T} & \cdots & h_{M i}^{T}\end{array}\right)^{T}$ is the length $L M$ vector of stacked channel coeffcients from the $M$ transmit antennas to the $i$ th receive antenna. Based on (2), the vector of all the received signals on all $N$ receive antennas can be expressed as $\operatorname{vec}(\boldsymbol{Y})=\left(\boldsymbol{I}_{N} \otimes S\right) \operatorname{vec}(\boldsymbol{H})+\operatorname{vec}(\boldsymbol{W})$, where the matrix $\boldsymbol{H}=\left(\begin{array}{lll}h_{1} & \cdots & h_{N}\end{array}\right)$.

\section{ORTHOGONAL BLOCK CODE DESIGN}

The orthogonality criterion for block codes for a frequencyselective channel is that the signal transmission matrix (code matrix) $S$ in (2) is orthogonal. Following the standard conventions for OSTBCs for ant-fading channels in $[6,12,14]$, we assume that the entries of the code matrix $S$ are $0, \pm s_{k}, \pm s_{k}^{*}$ or their multiples by $i=\sqrt{-1}$. The symbols $s_{k}, k=1,2, \cdots, q$ are taken from a complex signal constellation. The orthogonality constraint can be written as

$$
S^{H} S=\sum_{k=1}^{q}\left|s_{k}\right|^{2} I .
$$

The symbol rate of the code is $R=\frac{q}{T+L-1}$. Since $S$ has a block Toeplitz structure, the Hurwitz-Randon theory used in the design of OSTBCs for the atat-fading case can not be applied directly. However, we will show that the existing classes of orthogonal codes for tat-lading channels can be used to design orthogonal block codes for frequency-selective channels. Our analysis will start with the orthogonality among the sub-matrices in $S$. We then propose a two-step design scheme for orthogonal block codes for frequency-selective channels and establish the maximum symbol rate.

As indicated atter (2), the code matrix $S$ collects all the sig. nals from al] the transmit antennas; i.e., $S$ is composed of submatrices $S_{j}, j=1, \cdots, M$. Therefore, a necessary condition for $S$ to be orthogonal is that each $S_{j}$ is orthogonal. Let $s_{i, j}$ the $i$ th column of $S_{j}$. Because $S_{j}$ has a Toeplitz structure, the largest number of non-zero entries in the frst column of a matrix $S_{j}$ that is orthogonal cart be bounded, as we show in the following lemma. Furthermore, the zero entries have a certain pattern. This pattern plays an important part in our orthogonal design.

\subsection{Channel length $L=2$}

For convenience we will begin by detailing the design procedure for the case of $L=2$. The general case is summarized later.

Lemma 1 Given $T \geq 2$, the largest number of independent variables $x_{j} \in \mathbb{C}$ in the equation $\sum_{j=1}^{T-1} x_{j} x_{j+1}^{*}=0$ is $\left\lceil\frac{T}{2}\right\rceil$. One such arrangement is that in which the $x_{j}$ with even indices are zero and those with odd indices are free.

Proof: The proof will proceed by induction on $T$. The statement is immediate for $T=2$. For $T=3$, the largest number of independent non-zero variables for $x_{1} x_{2}^{*}+x_{2} x_{3}^{*}=0$ is 2 , which is achicved when $x_{2}=0$. For $T=4$, the largest number of independent variables for $x_{1} x_{2}^{*}+x_{2} x_{3}^{*}+x_{3} x_{4}^{*}=0$ is still 2 . Possible arrangements include the case where $x_{2}=x_{4}=0$. Thus the statement is true for $T=2 m+i, m=1, i=1,2$.

Suppose that for a given $K \geq 2$, the statement is true for $T=2 K+i . i=1,2$, i.e., the largest number of independent variables is $K+1$, and the non-zero variables have odd indices. For $T=2(K+1)+i, i=1,2$, we have

$$
\sum_{j=1}^{2(K+1)+i} x_{j} x_{j+1}^{*}=\sum_{j=1}^{2(K+1)} x_{j} x_{j+1}^{*}+\Delta,
$$


where $\Delta=\sum_{j=1}^{i} x_{2(K+1)+j-1} x_{2(K+1)+j}^{*}$. By the inductive hypothesis, $\sum_{j=1}^{2(\kappa+1)} x_{j} x_{j+1}^{*}=0$ and $x_{2 m}=0, m=$ $1,2, \cdots, K+1$. Therefore, to prove the statement for $T=$ $2(K+1)+i, i=1,2$, we need only consider $\Delta$. For the case where $i=1 . \Delta=x_{2(K+1)} x_{2(K+1)+1}$, but since $x_{2(K+1)}=0$. $\Delta=0$ independent of the value of $x_{2(K+1)+1}$. Therefore there are $K+2$ independent variables and the statement holds for $i=1$. For the case where $i=2, \Delta=x_{2(K+1)+1} x_{2(K+1)+2}$, where we have used the fact that $x_{2(K+1)}=0$. To ensure that $\Delta=0$ one of variables $x_{2(K+1)+1}$ and $x_{2(K+1)+2}$ must be zero. Hence, there are still $K+2$ independent variables and if we set $x_{2(K+1)+2}=0$ the free variables have odd indices and the even indexed variables are zero. Therefore, the statement holds for $i=2$ and the proof is complete.

Orthogonality of $S_{j}$ requires that $s_{1, j}^{H} s_{2, j}=0, j=$ $1, \cdots, M$. By applying Lemma 1 to this case, the non-zero entries for $s_{1, j}$ can be chosen as $\left\{s_{n, j} \mid n=1,3, \cdots, 2\left(\left\lceil\frac{T}{2}\right\rceil-1\right)+1\right\}$. Therefore, $S$ has the form.

$$
\left(\begin{array}{cccccccc}
s_{1} 1 & 0 & s_{1} 2 & 0 & \cdots & \cdots & s_{1} A & 0 \\
0 & s_{1}{ }_{1} & 0 & s_{1} & \cdots & \cdots & 0 & s_{1} M \\
s_{3} & 0 & s_{3} & 0 & \cdots & \cdots & s_{3} M_{1} & 0 \\
0 & s_{31} & 0 & s_{3} & \cdots & \cdots & 0 & s_{3} M \\
\vdots & \vdots & \vdots & \vdots & \cdots & \cdots & \vdots & \vdots \\
0 & s_{b 1} & 0 & s_{b} & \cdots & \cdots & 0 & s_{b} M
\end{array}\right)
$$

where $b=2\left\lceil\frac{T}{2}\right\rceil-1$. Now it can be seen from (6) that $S$ is orthogonal if and only if the frst columns among all $S_{j}$ 's orthogonal; i.c., if the matrix $G=\left(\begin{array}{llll}s_{1,1} & s_{1,2} & \cdots & s_{1, M}\end{array}\right)$ is orthogonal. Thus, the constraint for the orthogonality of $S$ can be decoupled into constraints for orthogonality in the delay and spatial domains. Therefore, an orthogonal design strategy can be implemented by the following two steps

- Step 1: Delay domain - Make the signal matrix for each transmit antenna, $S_{j}$, orthogonal.

- Step 2: Spatial domain - Make the frst signal vector among difterent transmit antennas orthogonal, i.e., make the matrix $G=\left(\begin{array}{llll}s_{1,1} & s_{1,2} & \cdots & s_{1, A}\end{array}\right)$ orthogonal

The maximum diversity gatn for a frequency-selective channcl is $M N L[3,15]$. That gain can be decomposed into the multipath diversity gain $L$ and spatial diversity gain $M N$. In our approach, the orthogonality constraint in Step I extracts the multipath diversity and that in Step 2 extracts the spatial diversity. Therefore, our approach provides full diversity, as expected. The design scheme leads to the following proposition.

Proposition 1 The maximum symbol rate of an orthogonal block code for a frequency-selective channel of length $L=2$ and $M$ transmit antenncts is $R=R_{\text {sust-mar }} / 2$, where $R_{\text {sutr-mar }}$ is the rate achieved by a rate-maximal orthogonal space time block code $Q$ for a bat fading channel with $M$ transmit anteninas. The code matrix $S=Q \otimes I_{2}$ achieves this rate, and the delay of that code is $d=2 d_{\text {D.J }}$, where $d_{\text {tur }}$ is the delay of $Q$.

Proof: By applying Lemma I, we need only consider code matrices of the form in (6). From the discussion above, the eftective size of $G$ is $\left\lceil\frac{T}{2}\right\rceil \times M$ if the zero entries are excluded. Suppose a code matrix $Q$ for a tat fading channel has a maxi-

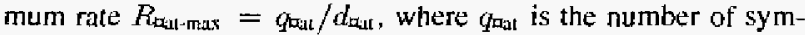
bols transmitted, and $d_{\text {sut }}$ is the delay. The corresponding $T$ for a frequency-selective channel is determined by $\left\lceil\frac{T}{2}\right\rceil=d$ xal. The minimum $T$ must be odd and is given by $T=2\left(d_{\text {out }}-1\right)+1$. Given that $L-1$ zeros are padded between the blocks in the frequency-selective case, the delay for a frequency-selective channel is $d=T+L-1=2 d_{h_{21}}$. Therefore, the maximal rate is $R=q_{\text {ata }} /\left(2 d_{\text {aat }}\right)=R_{\text {mal } \max } / 2$. The non-zero entries in the $£$ rst column in all $S_{j}$ 's can take the corresponding entries of $Q$. Due to the block Toeplitz structure of the code matrix $S$, it can be written as the Kronecker product of $Q$ and an identity of size of 2 . $S=Q \otimes I_{2}$

Note that the proof of Proposition 1 indicates that if the OSTBC used in the Kronecker product operation has minimum latency, the resulting orthogonal code for the frequency-selective channel also has minimum latency. Therefore, the minimum latency of an orthogonal code for $L=2$ is $2 d_{\text {sal-min }}$, where $d_{\mathrm{rat}_{\mathrm{a}} \text { min }}$ is the minimum delay of the OSTBC for the rat-fading case.

Let us examine some examples of Proposition 1 for systems with $L=2$ and different numbers of transmit antennas $M$. For $M=2$, the minimum block length is $T=3$. The code matrix $S=\left(\begin{array}{ll}S_{1} & S_{2}\end{array}\right)=\left(\begin{array}{llll}s_{1,1} & s_{2,1} & s_{1,2} & s_{2,2}\end{array}\right)$. The orthogonality constraint in delay domain (Step I) makes $S_{1}$ and $S_{2}$ orthogonal. Hence, after Step 1, we have

$$
S_{\mathrm{st}_{1}}^{(2,2)}=\left(\begin{array}{cc:cc}
s_{1} t_{1} & 0 & s_{12} & 0 \\
0 & s_{11} & 0 & s_{12} \\
s_{1} 1 & 0 & s_{32} & 0 \\
0 & s_{31} & 0 & s_{32}
\end{array}\right)
$$

where the superscript ${ }^{(2,2)}$ represents $M=2$ and $L=2$. The role of Step 2 is to make the frst columns in $S_{1}$ and $S_{2}$ orthogonal, i.e., to make the matrix $G=\left(\begin{array}{ll}s_{1,1} & s_{1,2}\end{array}\right)$ orthogonal. If the zero entries are excluded, $G$ has an effective size of $2 \times 2$. The maximum number of symbols that can be transmitted is 2 , and this rate is achieved by Alamouti's scheme [1]. The delay in this case is $d=T+L-1=4$, the rate is $2 / 4=1 / 2$, and the code matrix can be constructed as

$$
S^{(2,2)}=\left(\begin{array}{cccc}
s_{1} & 0 & s_{2} & 0 \\
0 & s_{1} & 0 & s_{2} \\
-s_{2}^{*} & 0 & s_{1}^{*} & 0 \\
0 & -s_{2}^{*} & 0 & s_{j}^{*}
\end{array}\right)=\left(\begin{array}{cc}
s_{1} & s_{2} \\
-s_{2}^{*} & s_{1}^{*}
\end{array}\right) \otimes I_{2} .
$$

For the case of $M=4$ antennas at the transmitter, the minimum block length is $T=7$. The maximum symbol rate of an orthogonal code is $3 / 8$, and a rate-maximal code is

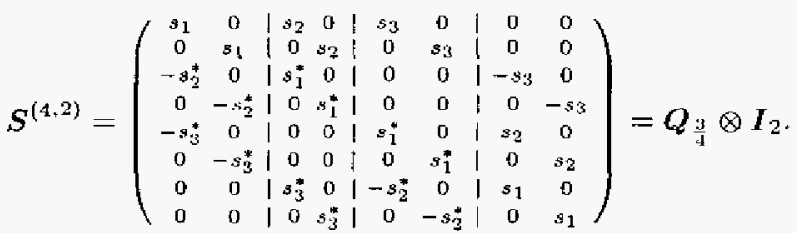

where $Q_{\frac{3}{4}}=\left(\begin{array}{cccc}s_{1} & s_{2} & s_{3} & 0 \\ -s_{2}^{*} & s_{1}^{*} & 0 & -s_{3} \\ -s_{2}^{*} & 0 & s_{1}^{*} & s_{2} \\ 0 & s_{3} & -s_{2} & s_{1}\end{array}\right)$, is the $3 / 4$ OSTBC for a aat fading channel [12-14]. Observe that the codes $S^{(2,2)}$ in (8) and $S^{(4,2)}$ in (9) not only have the maximum rate but also have minimum latency, since Alamouti's scheme and $Q_{\frac{3}{4}}$ have the min:mum latency. For the case of $M=3$, an orthogonal code can be obtained from the $M=4$ case in (9) by removing 2 columns, say the tast two columns. The maximal symbol rate remains the same.

For $M=5$ and 6 transmit antennas, the maximum rate of an OSTBC for a tading channel is $2 / 3$, and this can be achieved with a with code length of 15 [6]. Applying the $2 / 3$ code to our 
design approach, the maximum orthogonal code rate for $L=2$ is $R=1 / 3$ with delay of 30 . The code has the form

$$
S^{(5,2)}=Q_{\frac{2}{3}} \otimes I_{2}
$$

where $Q_{\frac{2}{3}}$ is given in [6]. The code $S^{(5,2)}$ in (10) has the maximum rate but not minimum latency because $Q_{\frac{2}{3}}$ has the maximum rate but not mirimum latency. The minimum latency of OSTBC for 5 or 6 transmit antennas in the atat-fading case is $2^{\left[\log _{2} M ?\right.}=8,[14]$. Therefore, the minimum latency of the orthogonal code for $M=5,6$ and $L=2$ is 16 , rather than the delay of 30 required by the code in (10). However, our design scheme can be applied to construct a minimal-iatency orthogonal code for frequency-selective charnels of $L=2$ by simply using the minimum latency OSTBCs described in [14] in the Kronecker product. (The resulting codes are not necessarily rate maximal if $M \geq 5$.)

It is also possible to construct a non-maximal-rate and nonminimal-latency orthogonal block code using our design procedure. For example, for $L=2$ and $M=4$ a rate $1 / 4$ orthogonal block code can be constructed as $Q_{\frac{1}{2}} \otimes I_{2}$, where $Q_{\frac{1}{2}}$ is the rate $1 / 2$ OSTBC given in [13].

\subsection{General case}

For a frequency-selective channel with length $L \geq 3$, our results can be generalized to the following lemma and proposition. The proof of the lemma is given in the Appendix, and that of the proposition is analogous to the proof of Proposition $\mathbf{I}$.

Lemma 2 Given $T \geq L$, the largest number of independent variables $x_{j} \in \mathbb{C}$ in the $L-1$ equations

$$
\sum_{j=1}^{T-\ell} x_{j} x_{j+\ell}^{*}=0 \quad \ell=1,2, \cdots, L-1,
$$

is $\left\lceil\frac{T}{L}\right\rceil$. The free variables can be $x_{1}, x_{L+1}, \cdots, x_{L\left(\left\lceil\frac{T}{L}\right\rceil-1\right)+1}$.

Proposition 2 The maximum symbol rate of an orthogonal block code for a frequency-selective channel of length $L$ and $M$ transmit antennas is $R=R_{\mathrm{Ear-max}} / L$, where $R_{\text {Dur-mar }}$ is the rate achieved by a maximal rate orthogonal space time block code $Q$ for a a at fading channel with $M$ iransmit antennas. The code matrix $S=$ $Q \otimes I_{L}$ achieves this rate, and the delay of that code is $d=L d_{\text {at }}$, where $d_{\text {xal }}$ is the delay of $Q$.

Analogous to the case where $L=2$, the minimum latency of the orthogonal code is $L d_{\text {ant }}$-min -

\section{SIMULATION}

We consider a system with 2 transmit antennas and 1 reccive antenna. The channel is a frequency-selective Rayleigh fading channel with memory length 2 . We compare the proposed orthogonal code with the ST-OFDM and CGDD schemes. Each scheme is allocated the same transmission power and we consider schemes with a symbol block length of 4 . Using Alamouti's code [1], the ST-OFDM and CGDD schemes $[3,5]$ will require 5 channel uses, and these schemes have a symbol rate of $4 / 5$. In contrast. the proposed scheme has a symbol rate of $1 / 2$.

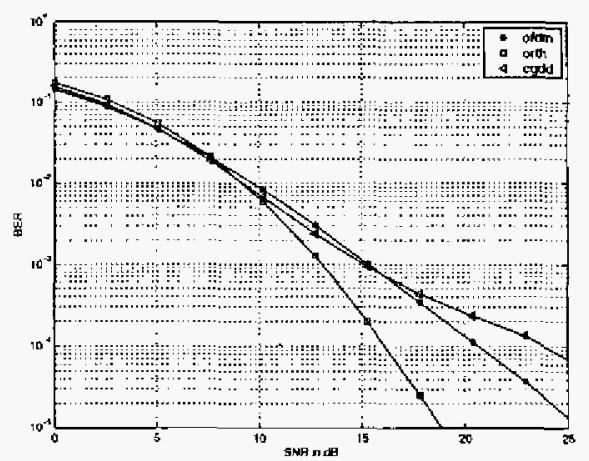

Fig. 1. BER performance against block SNR for our orthogonal design (at 1 bits per channel use) and the ST-OFDM and CGDD schemes (at $4 / 5$ bits per channel use).

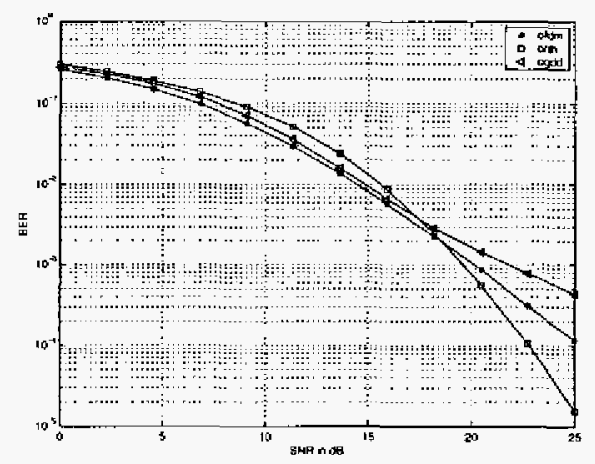

Fig. 2. BER performance against block SNR for our orthogonal design (at 2 bits per chamel use) and the ST-OFDM and CGDD schemes (at $8 / 5$ bits per channel use).

In Fig 1 we have provided the BER performance of the proposed scheme when 4-QAM signalling is used. In this case the bit rate is 1 bit per channel use. In that fgure we have also plotted the BER pertormance of the ST-OFDM and CGDD schemes with BPSK signalling, which results in a slightly lower bit rate of $4 / 5$ bits per channel use. In Fig 2 we have provided the BER performance of the proposed scheme with 16-QAM signalling (2 bits per channel use) and that of the ST-OFDM and CGDD schemes with 4-QAM signalling ( $8 / 5$ bits per channcl use).

Despite the fact that these confgurations result in the proposed scheme having a higher data rate than its competitors. the simulation results demonstrate improved performance at moderate-tohigh SNRs. This is due to the fact that the proposed scheme provides full diversity. Actually, the performance advantage of the proposed scheme is slightly larger than shown, because we have neglected the power used to transmit the cyclic prefx in the calculation of the SNR for the ST-OFDM and CGDD schemes.

\section{CONCLUSION}

A direct construction of orthogonal block codes for frequencyselective multiple antenna channels has been derived. The maximum achievable (symbol) rate was found and a rate-maximizing 
code structure was specifed. The orthogonal design can be realized by a two-step orthogonality strategy. The frst step is to make the signal matrix transmitted from each antenna self-orthogonal. This step obtains delay diversity. The second step is to make the Erst signal vector among all transmit antennas orthogonal. This step obtains spatial diversity. For a aat-fading channel only the second step needs to be considered. This two-step design strategy reveals the relationship between the orthogonal block codes for frequency-selective channels and those for aat-fading channels. An orthogonal block code for a frequency-selective channel of memory length of $L$ can be simply constructed by the Kronecker product of an existing orthogonal space time block code matrix for the aat-fading channels with an identity matrix of size of $L$. If the orthogonal space-time block code for the ant-fading channels used in the Kronecker product operation is rate maximal or latency minimal, the resulting orthogonal code for the frequency-selective channels is rate maximal or latency minimal, respectively.

Acknowledgement: The authors wish to thank Dr. ZhiQuan (Tom) Luo of The University of Minnesota and Dr. JianKang Zhang of McMaster University for valuable discussions.

\section{APPENDIX: PROOF OF LEMMA 2}

For the case of $L=2$, the statement collapses to that in Lemma 1 , the proof of which involved induction on $T$. To prove the more general statement in Lemma 2, we will employ an additional (outer) induction on $L$.

The (outer) inductive hypothesis that the statement holds for $L=P$ states that for $T \geq P$ there are $\left\lceil\frac{T}{P}\right\rceil$ free variables in

$$
\left\{x_{j} \mid \mathcal{S}_{T, \ell} \stackrel{\Delta}{=} \sum_{j=1}^{\gamma-\ell} x_{j} x_{j+\ell}^{*}=0, \ell=1,2, \ldots, P-1\right\}_{j=1}^{T}
$$

and that they may be chosen to be $x_{1}, x_{P+1}, \cdots, x_{\left.P\left(1, \frac{T}{T}\right]-1\right]+1}$. To show that this hypothesis implies that when $L=P+1$ the statement holds for all $T \geq P+1$ we will use a structured induction argument on $T$. We will treat the case of $T=P+1$ separately, and then will use induction on $K$ and $i$ to show that the lemma holds for $T=K(P+1)+i$ for all $K \geq 1$ and $1 \leq i \leq P+1$.

For $T=P+1$ we have that $\mathcal{S}_{P+1, \ell}=S_{P, \ell}+\Delta_{P+1, \ell}$, where $\Delta_{P+l, \ell}=x_{P+1-\ell} x_{P+1}^{*}$. By the (outer) inductive hypothesis (on $L$ ) we have that $S_{P, \ell}=0$ and $\Delta_{P+1, \ell}=0$ for all $1 \leq \ell \leq P-1$. Therefore, we need only enforce $\mathcal{S}_{F^{\prime}+1, P}=x_{1} x_{P+1}^{*}=0$. To do so, we can simply set $x_{P+1}=0$, which results in one free variable, $x_{1}$, as stated in the lemma.

For $T=P+2$, we have $S_{P+2, \ell}=S_{P+1, \ell}+\Delta_{\ell}$, where $\Delta P+2, \ell=x_{P+2-\ell} x_{P+2}^{*}$. Under the (outer) inductive hypothesis (on $L$ ), $S_{P+1, \ell}=0$ for $1 \leq \ell \leq P-1$ and $\Delta_{P+2, \ell}=0$ for $2 \leq \ell \leq P$. Therefore, the equations which remain to be satisfed are $\mathcal{S}_{P+1, P}=x_{1} x_{P+1}^{*}=0$ and $\Delta_{P+2,1}=x_{P+1} x_{P+2}=0$. By setting $x_{P+1}=0$ we satisfy both equations and retain the largest number of free variables. Hence the lemina holds for $T=P+2$. We now make the (inner) hypothesis that the lemma holds for $T=$ $P+1+i$ for some $1 \leq i \leq P$, and examine the equations when $T=P+2+i$. Under this hypothesis, $\mathcal{S}_{P+2+i, \ell}=0$, except when $\ell=i$. When $\ell=i$ we have $\mathcal{S}_{P+2+i, i}=x_{P+2} x_{P+2+i}^{*}$, and hence we must choose $x_{P+2+i}=0$ in order to satisfy (li). We have now shown that if the lemma holds for $L=P$ then it holds for $L=P+1$ and $P+1 \leq T \leq 2(P+1)$. What remains to be shown is that the lemma also holds for larger values of $T$.

Suppose that for some $K \geq 2$ the statement holds for $L=$ $P+1$ and $T=K(P+1)+m$, for all $1 \leq m \leq P+1$. and consider the case where $T=(K+1)(P+1)+i$, for $1 \leq i \leq$ $P+1$. For $i=1$ we have $\mathcal{S}_{(K+1)(P+1)+1, \ell}=\mathcal{S}_{(K+1)(P+1), \ell}+$ $x_{(P+1)(K+1)+1-\ell} x_{(K+1)(P+1)+1}^{*}$. The inductive hypothesis on $K$ ensures that $\mathcal{S}_{(K+1)(P+1), \ell}=0$ and $x_{(K+1)(P+1)+1-\ell}=0$ for all $1 \leq \ell \leq P$. Hence, $x_{(K+1)(P+1)+1}$ may be chosen freely, and the lemma holds. If we assume that the lemma holds for $T=$ $(K+1)(P+1)+i$ for some $1 \leq i \leq P$, then for $T=(K+$ 1) $(P+1)+i+1$ we have that $S_{(K+1)(P+1)+i+1, \ell}=0$. except when $\ell=i$. Therefore, in order to satisfy (11) we must choose $x_{(K+1)(P+1)+i+1}=0$. Hence, the lemma holds for $T=(K+$ 1) $(P+1)+i, 1 \leq i \leq P+1$. These induction arguments on $K$ and $i$ verify that the outer induction argument on $L$ holds for all values of $T \geq 2(P+1)+1$, and hence the proof is complete.

\section{REFERENCES}

[1] S. M. Alamouti, "A Simple Transmit Diversity Technique for Wireless Communications," IEEE J. Select. Areas Commun., vol. 16, pp. 145I-1458, Oct. 1998.

[2] H. El Gamal and M. O. Damen, "Linear Threaded Algebraic SpaceTime constellations," IEEE Trans. Informat. Theory, pp. 2372 23868, Oct. 2002.

[3] D. Gore, S. Santhu and A. Paulraj, "Delay Diversity Codes for Frequency Selective Channels," in Pros: Int. Conf. Commmn., vol. 3. pp. 1948-1953,2002.

[4] E. G. Larsson, P. Stoica, E. Lindskog and 3. Li, "A Space-Time Block Coding for Frequency-Selcctive Channels," in Proc: Int. Conf. Aconstics, Speech, Signal Processing, vol. 3. pp. 2405-2409, 2002.

[5] K. F. Lee and D. B. Williams, "A Space-Time Coded Transmitter Diversity Technique for Frequency selective Fiding Channcls", in IEEE Sensor Array \& Multichannel Signal Processing Whshp, pp. 149-152, Mar. 2000.

[6] X.-B. Liang, "Orthogona] Designs With Maximal Rittes," IEEE Tons. Informat. Theory, vol. 49, pp. 2468-2503, Oct. 2003.

[7] Y. Liu, M. P. Fitz, O. Y. Takeshita, "Space-time Codes Performance Criteria and Design for Frequency Selective Fading Channe[s," in Proc: IEEE Int. Conf. Commum., vol. 9, pp. 2800-2804, 2001.

[8] X. Ma and G. B. Giannakis, "Full-rate Full-diversity Complex-feld Space-time Codes for Frequency- or Time-selective Fading Channels," in Proc. Asilomar Conf. on Signals, Syitems and Computers, Vol 2. pp. 17 [4-1718, 2000.

[9] P. Stoica and E. Lindskog, "Space-Tine Block Coding for Channels with Intersymbol Interfertnce," Digital Signal Processing. vol. 12, pp. 616-627, Dec. 2002

[10] W. Su and X.-G Xia, "On Space-Time Block Codes from Complex Orthogonal Designs," Wireless Personal Commun., vol. 25, pp. 1-26, Apr. 2003.

[11] W. Su, Z. S. Masoud, and K. J. R. Liu. "Obtaining Full-Diversity Space-Frequency Codes from Space-Time Codes via Mapping," IEEE Trans. Signal Processing, vol 51, pp. 2905-2916, Nov. 2003.

[12] V. Tarokh and A. R, Calderbank. "Space.Time Block Codes from Orthogonal Designs," IEEE Trants. Informat. Theory, vol. 45, pp. 1456-1467, July 1999.

[13] V. Tarokh and A. R. Calderbank, "Space-Time Codes for High Data Rate Wireless Communication: Performance Criterion and Code Construction," IEEE Trans. Commut., vol. 47, pp. 199-207, Feb. 1999.

[14] O. Tirkkonen and A. Hottinen, "Square-Matrix Embeddable SpaceTime Block Codes for Complex Signal Constellations." IEEE Trans. Informat. Theory, vol. 48, pp.384-395, Feb. 2002.

[15] S. Zhou and G. B. Giannakis, "Single-Carrier Space-Time BlockCoded Transmissions Over Frequency-Selective Fading Channels." IEEE Trans. Informat. Theory, vol 49, pp. 164-179, Jan. 2003. 